\title{
Manual Tracking Performance in Patients with Cerebellar Incoordination: Effects of Mechanical Loading
}

\author{
Betty-Lynn Morrice, Werner J. Becker, J.A. Hoffer and Robert G. Lee
}

\begin{abstract}
Manual tracking performance was studied in five patients with cerebellar incoordination due to unilateral cerebellar hemisphere lesions. The subjects were required to track a target on an oscilloscope screen by moving a cursor controlled by flexion-extension movements of the wrist. In comparison to normal subjects, the cerebellar patients, using their clinically affected arm, demonstrated irregular tracking patterns with inappropriate accelerations and decelerations, numerous high velocity peaks of movement, and an increased time lag between the cursor and the target.

The addition of a viscous load provided by feeding back wrist velocity to a torque motor coupled to the apparatus resulted in significant improvement in tracking performance and suppression of the high velocity peaks. Increasing elastic stiffness by feeding back wrist position or inertial load by adding weights to the hand did not improve performance on this task. It is proposed that a hypotonic cerebellar limb behaves like an underdamped mechanical system. The addition of viscous loads helps restore more normal damping during voluntary movements of the arm.

RÉSUMÉ: Poursuite manuelle chez les patients présentant une incoordination cérébelleuse: effet d'une surcharge mécanique La poursuite manuelle a été étudiée chez cinq patients atteints d'incoordination cérébelleuse due à des lésions unilatérales d'un hémisphère cérébelleux. Les sujets devaient poursuivre une cible sur un écran d'oscilloscope en déplaçant un curseur contrôlé par des mouvements de flexion et d'extension du poignet. Par rapport à des sujets normaux, les patients avec atteinte cérébelleuse exécutaient des tracés de poursuite irréguliers avec des accélérations et décélérations inappropriées, de nombreuses pointes de mouvement de haute vélocité et une augmentation du délai entre le curseur et la cible. L'ajout d'une charge visqueuse, provenant de la vélocité du poignet agissant sur un moteur couplé à l'appareillage, a résulté en une amélioration significative dans l'exécution de la poursuite et une suppression des pointes de haute vélocité. Ni une augmentation de la rigidité élastique produite par la localisation de la position du poignet, ni une augmentation de la charge d'inertie produite par l'ajout de poids à la main n'ont amélioré l'exécution de la tâche. Nous proposons qu'un membre présentant une hypotonie cérébelleuse se comporte comme un système mécanique sous-amorti. L'addition de charges visqueuses aide à rétablir un amortissement plus normal pendant les mouvements volontaires du bras.
\end{abstract}

Can. J. Neurol. Sci. 1990; 17:275-285

Lesions involving the lateral parts of the cerebellar hemispheres and associated deep nuclei produce characteristic abnormalities in control of limb movement. 1,2 Some of these deficits such as decomposition of movement are only apparent during complex multi-joint movements involving an entire limb. Other features of cerebellar dysfunction are obvious during simple movements involving a single joint, and can be studied by having subjects track a moving visual target with a cursor controlled by the hand or arm. 3.,5 In this type of task, patients with cerebellar dysfunction have difficulty generating smooth tracking movements. Their tracking patterns show a series of small steps or "saccades". The appearance is similar to the phenomenon of sac- cadic interruption of pursuit eye movement which may also occur as a result of damage to certain parts of the cerebellum. ${ }^{6}$ Similar discontinuities of arm movement have been described in monkeys during cooling of the deep cerebellar nuclei.?

It is a common clinical observation that patients with cerebellar limb incoordination have particular difficulty controlling movement velocity. Velocities are often inappropriately high for the task being performed. This is obvious when patients are required to track a slowly moving visual target and it may also be partially responsible for the overshoot which often occurs when subjects with cerebellar damage are required to move a finger towards a target in space. A similar phenomenon with high

From the Department of Clinical Neurosciences, University of Calgary, Calgary, Alberta, Canada

Received March 6, 1990. Accepted April 9, 1990

Reprint requests to: Dr. Robert G. Lee, Department of Clinical Neurosciences, Foothills Hospital, 1403 - 29th Street N.W., Calgary, Alberta, Canada T2N 2T9 
velocity peaks has been demonstrated in monkeys with reversible cerebellar dysfunction produced by cooling the cerebellum. ${ }^{8}$ One factor which may contribute to the difficulty controlling movement velocity is the hypotonia which is commonly associated with cerebellar dysfunction. Holmes ${ }^{2}$ considered hypotonia to be one of the fundamental deficits resulting from cerebellar damage and felt that it might be responsible for other abnormalities such as dysmetria and postural instability. Normal muscle tone may provide sufficient viscous stiffness to dampen inappropriately high velocity movements and to prevent the limb from overshooting a target. Reduced tone may cause a limb to behave like an underdamped mechanical system. A characteristic feature of such a system is a tendency to develop oscillations.

In this study we investigated whether manual tracking performance in cerebellar patients can be improved by modifying the mechanical properties of the manipulandum being used for tracking. Specifically, we examined the effects of increasing viscous stiffness, elastic stiffness, and inertial load. It was found that errors in tracking performance were reduced most consistently by increasing the viscous stiffness of the system.

\section{Methods}

\section{Subjects}

Five patients ( 4 females, 1 male; age range 16-30) with predominantly unilateral lesions involving one cerebellar hemisphere were selected for these experiments. The clinical details are summarized in Table 1. Four of the patients had undergone resection of a cerebellar tumour; the fifth patient had a cerebellar infarct. CT scans were reviewed to confirm the site and extent of the cerebellar lesion. All patients were studied at least several months after their initial presentation at a time when the neurologic deficit had become stable. The clinical abnormalities included dysmetria, incoordination, hypotonia, and slowing and irregularity of rapid alternating movements involving the arm ipsilateral to the lesion. None of the patients had prominent intention tremor. The cerebellar deficit ranged in severity from mild to moderate, and all patients were independent in activities of daily living. In each patient, tracking performance was studied in both arms. The arm contralateral to the lesion did not show clinical signs of cerebellar dysfunction in any of the five patients. In addition, six healthy control subjects were studied. Their age range (14-29) was comparable to that of the patients. Informed consent was obtained from all subjects following an explanation of the purpose of the experiment and the procedures involved.

\section{Experimental procedure}

Subjects were seated comfortably with one forearm supported in a manipulandum that restricted movement to the wrist joint. A vertical rod coupled the manipulandum to a torque motor (Aeroflex TQ $82 \mathrm{~W}$ ) that was used to provide resistive loads. The arm was positioned so that the wrist joint was located directly along the axis of rotation of the manipulandum. Wrist angle or position was monitored by a precision potentiometer attached to the vertical rod, and the signal from the potentiometer was differentiated to obtain wrist velocity. The subjects were presented with a visual display on a CRT screen situated at eye level 1 metre directly in front of the subject. The display consisted of a $2 \mathrm{~cm}$ open square target and a $1.25 \mathrm{~cm}$ shaded square cursor that the subject controlled by moving the wrist joint. The target moved horizontally back and forth across the screen in a complex pattern that was created by summing two sinusoidal waves with frequencies of $0.15 \mathrm{~Hz}$ and $0.345 \mathrm{~Hz}$.

The subjects were instructed to keep the cursor aligned with the target. This required carefully controlled flexion-extension movements at the wrist joint over an angular distance of 35 degrees. Target velocities ranged from $0-30$ degrees per second. Each trial lasted 20 seconds. To minimize effects of learning, the trials commenced at different points in the stored target pattern. Target movements and data collection were controlled by a PDPI1-40 computer. The output from the potentiometer was sampled at a rate of $100 \mathrm{~Hz}$.

A viscous load was provided by feeding back the velocity of handle movement to the amplifier that controlled the torque motor. Similarly, an elastic load was provided by feeding back handle position to the torque motor. The inertial load was increased by attaching weights to the subject's hand. In addition to unloaded trials, each subject performed the tracking task with two levels of viscous stiffness $(0.022$ and 0.044 Newtons/ $\mathrm{sec} / \mathrm{degree})$, two levels of elastic stiffness ( 0.44 and 0.93 Newtons/degree) and two inertial loads $(0.5 \mathrm{~kg}$ and $1.0 \mathrm{~kg})$. Although it would have been optimal to study the effects of a large number of different loads over a wide range, this would have made the experiment unacceptably long for the subjects. We therefore made an arbitrary selection of a low and a high load for each of the three conditions. A standard experiment consisted of a total of 54 twenty second tracking trials with a short rest period after each trial. The initial 10 trials were performed without any added load to allow the subjects to familiarize themselves with the apparatus and procedure. Following this they performed 6 trials for each of the 6 loaded conditions. The different mechanical loads were presented in random order. Following each set of 6 trials with one of the loaded conditions, the subjects performed three unloaded trials.

\section{Data analysis}

For each 20 second trial a positional error score was calculated by summing the absolute values of the point-by-point

Table 1: Type of Pathology and Clinical Findings in Patients with Unilateral Cerebellar Lesions

\begin{tabular}{|c|c|c|c|c|c|c|c|}
\hline $\begin{array}{l}\text { Patient } \\
\text { Number }\end{array}$ & Age & Sex & Pathology & Dysmetria & $\begin{array}{l}\text { Rapid Alternating } \\
\text { Movement }\end{array}$ & $\begin{array}{l}\text { Intention } \\
\text { Tremor }\end{array}$ & Tone \\
\hline 1 & 29 & $\mathrm{~F}$ & $\begin{array}{l}\text { Posterior fossa epidermoid tumour extending } \\
\text { into right cerebellar hemisphere }\end{array}$ & mild & moderate impairment & absent & decreased \\
\hline 2 & 30 & M & $\begin{array}{l}\text { Hemangioblastoma right cerebellar } \\
\text { hemisphere }\end{array}$ & mild & normal & absent & normal \\
\hline 3 & 20 & $\mathrm{~F}$ & Astrocytoma right cerebellar hemisphere & moderate & moderate impairment & mild & normal \\
\hline 4 & 16 & $\mathrm{~F}$ & Astrocytoma left cerebellar hemisphere & moderate & moderate impairment & mild & decreased \\
\hline 5 & 25 & $\mathbf{F}$ & Infarct right cerebellar hemisphere & mild & mild impairment & absent & normal \\
\hline
\end{tabular}


difference between target and cursor positions over the 2000 data points. The data were analyzed initially using an analysis of variance (ANOVA) to compare error scores during all sets of unloaded and loaded trials in each subject and to compare between each of the three data groups (control subjects, clinically normal arms of patients, and affected arms). Because the patients represented a heterogeneous group with considerable variation in the degree of cerebellar incoordination, additional analysis was carried out to examine the specific effects of the different types of mechanical loading in each individual subject. Mean positional error scores for each set of six trials with one of the resistive loads were compared to the mean for the total of six unloaded trials immediately preceding and following the loaded trials, using Student's t-test.

The velocity records were analyzed in a similar manner to obtain a velocity error score. Movement velocity was also analyzed by measuring the number of velocity peaks exceeding 30 degrees/sec. and the number of baseline crossings in the velocity trace.

\section{Additional studies to investigate the role of vision}

Visual reaction times for the type of motor task used in this study were determined by measuring the latency from sudden movement of the visual target to onset of wrist flexion. In addition, the average time lag between target and hand movement for individual $20 \mathrm{sec}$. tracking trials was computing by perform- ing sequential time shifts of all the data points representing cursor position with respect to target position. The positional error score was re-calculated following each time shift of the data points until a point was identified at which the positional error score reached a minimal value. The tracking delay, or amount of time shift required to minimize positional error, was calculated for both the clinically affected and normal arms of the patients and results were compared with control subjects.

To determine whether inappropriate corrections for visually detected errors might be contributing to the high velocity irregular movements during the tracking tasks, wrist movements with and without visual feedback were compared. For these experiments, the target moved in a regular sinusoidal pattern rather than in the unpredictable pattern used in the previous studies. After ten initial trials with visual feedback of target and cursor, the display was removed and subjects were instructed to carry out the same sequence of wrist flexion-extension movements without any visual feedback.

\section{Results}

\section{Tracking performance without external loading}

None of the cerebellar patients were able to generate a smooth tracking pattern with their affected arm. The position recordings showed numerous irregularities with inappropriate accelerations, decelerations and reversals in direction. As a result,

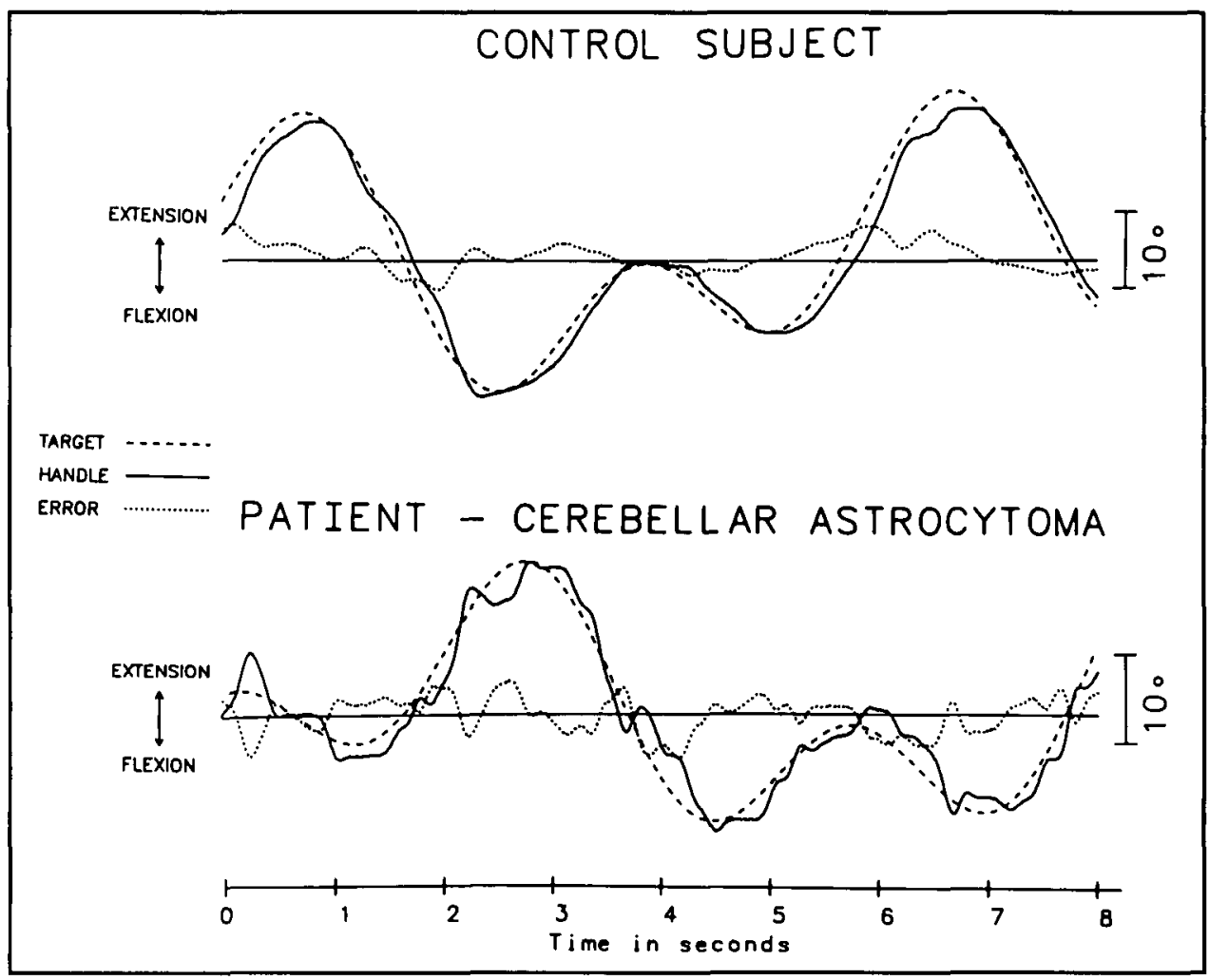

Figure I - Tracking performance without any added load in a control subject and in a cerebellar patient using the clinically affected arm. A representative $8 \mathrm{sec}$ segment of the $20 \mathrm{sec}$ trial is shown. The dashed line represents target movement; the solid line is the position of a cursor controlled by the subject performing flexion-extension movements at the wrist. The fine dotted line represents the instantaneous error obtained by taking the difference between target and cursor position for each data point (sampling rate 100/sec). The cumulative 20 second error scores in these examples, expressed in arbitrary units, were 39508 for the control subject and 65052 for the patient. 


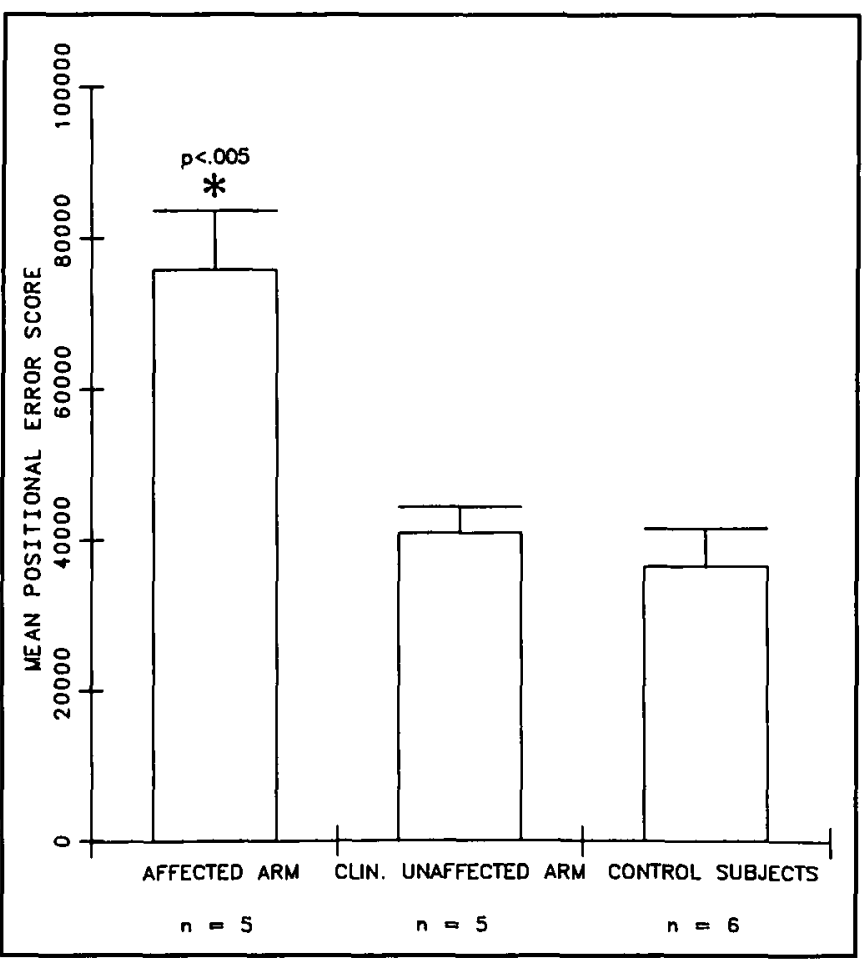

Figure 2 - Mean positional error scores and standard errors for six control subjects and five cerebellar patients. Values for both the affected and climically normal arms of the patients are shown. Results for each subject were obtained by averaging the cumulative error scores for all 28 trials performed without added mechanical loads. positional error scores were much higher than in the control subjects. Figure 1 shows representative segments of the position records for a normal subject and for patient with persisting cerebellar incoordination following removal of a cerebellar astrocytoma. In these examples, the total positional error scores for the twenty second trial, represented by the fine dotted line in Figure 1, were 65752 for the patient and 39508 for the control subject. These values are arbitrary units obtained by calculating the difference between the target position and cursor position for each data point.

For each subject the mean positional error score was determined by averaging the cumulative error scores for all 28 trials performed without added loads. These results are illustrated in Figure 2. ANOVA showed that within each of the three data groups there was no difference between the seven sets of unloaded trials; therefore, this mean positional error score could be used for comparison with the error scores during the loaded conditions. The mean error score for the affected arms of the five

Table 2: Analysis of Tracking Velocities for Control Subjects and Cerebellar Patients

\begin{tabular}{|c|c|c|c|}
\hline & \multirow[b]{2}{*}{ Controls } & \multicolumn{2}{|c|}{ Patients } \\
\hline & & $\begin{array}{c}\text { Unaffected } \\
\text { Arm }\end{array}$ & $\begin{array}{c}\text { Affected } \\
\text { Arm }\end{array}$ \\
\hline Velocity error score & $17234 ! \pm 1283$ & $19485 \pm 667$ & $36918 \pm 13742$ \\
\hline $\begin{array}{l}\text { Number of velocity } \\
\text { peaks with amplitude } \\
>30 \text { degrees/sec }\end{array}$ & $6.69 \pm 0.95$ & $8.58 \pm 0.75$ & $19.13 \pm 0.50^{2}$ \\
\hline $\begin{array}{l}\text { Number of zero } \\
\text { crossings }\end{array}$ & $2.69 \pm 0.66$ & $5.79 \pm 2.51$ & $23.21 \pm 4.63^{3}$ \\
\hline
\end{tabular}

1 Mean values $\pm S E$ for all trials without added loads

$2 p<0.005$ with respect to controls

${ }^{3} p<0.01$ with respect to controls

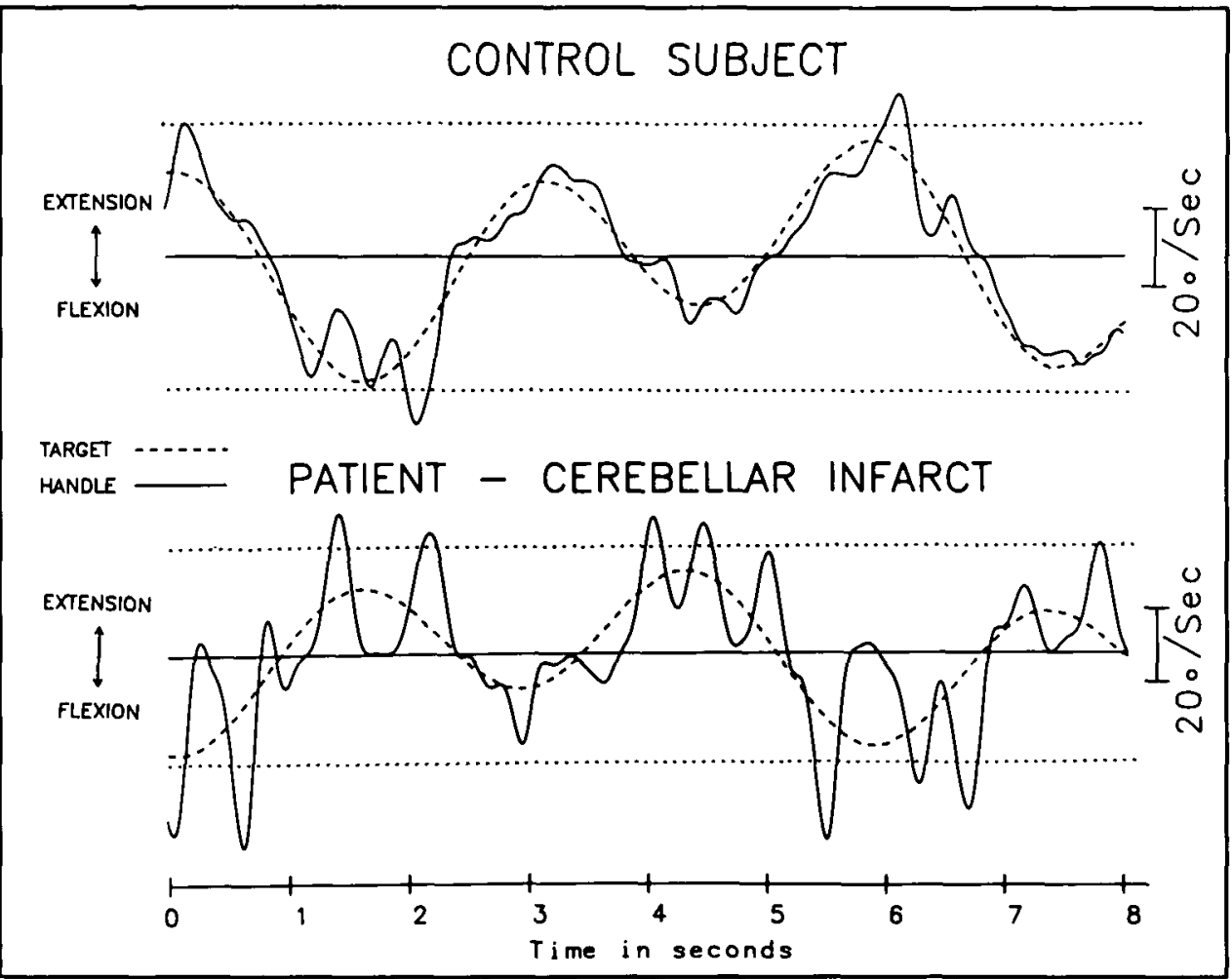

Figure 3 - Velocity tracings for the target and for the subject's cursor during a 8 second segment of a $20 \mathrm{sec}$ ond tracking trial without added mechanical load. Resulss are shown for a control subject and for a patient with incoordination of one arm due to a cerebellar infarct. Velocity was obtained by differentiating the signal from the potentiometer representing wrist position. The horizontal dotted lines indicate velocities of $\pm 30 \% \mathrm{sec}$ In the cerebellar patient velocity peaks commonly exceed this level. 
cerebellar patients was 75998 . This value is approximately twice as large as those obtained from the clinically unaffected arms of the patients and from the six control subjects. A Tukey HSD procedure showed no difference between mean positional error scores for the unaffected arms of the patients and for the normal subjects during unloaded trials. Error scores for the patients' affected arms were significantly higher.

The abnormal features in tracking performance in the cerebellar patients were clearly revealed when the position records were differentiated to examine the instantaneous velocity of movement. Typical movement velocity traces during a portion of the tracking task are shown in Figure $\mathbf{3}$ for a control subject and for a patient with a unilateral cerebellar lesion. The recording from the patient shows numerous high velocity peaks. The number of peaks exceeding $30 \mathrm{deg} / \mathrm{sec}$ during each 20 second trial were counted. In normal subjects peak velocities exceeded this level only occasionally whereas this was a frequent observation in patients with cerebellar incoordination (Table 2). Irregularities in movement velocity were also reflected by an increase in the number of zero crossings. Mean values for these measurements for patients and control subjects are also presented in Table 2.

\section{Effects of added loads}

The addition of a viscous load produced a marked improvement in the tracking performance of the clinically affected arm in all five cerebellar patients. Figure 4 shows position and velocity traces without loading and with a viscous load during representative tracking trials by one of the cerebellar patients. Without loading the positional error score for this twenty second trial was 67629; with a viscous load the error score was reduced to 28142 . The added viscosity reduced the velocily error score from 38355 to 13410 .

When the results from all experiments in control subjects and cerebellar patients were examined using ANOVA, differences between loaded and unloaded conditions were identified for the controls and for the affected arms of the patients. For the clinically unaffected arms, no significant change in error score was noted with any of the loaded conditions when results were pooled from all patients.

To determine more precisely which conditions produced a change and how the different mechanical loads affected tracking performance in individual subjects, the data were analyzed using paired t-tests. In the patients a high viscous resistance

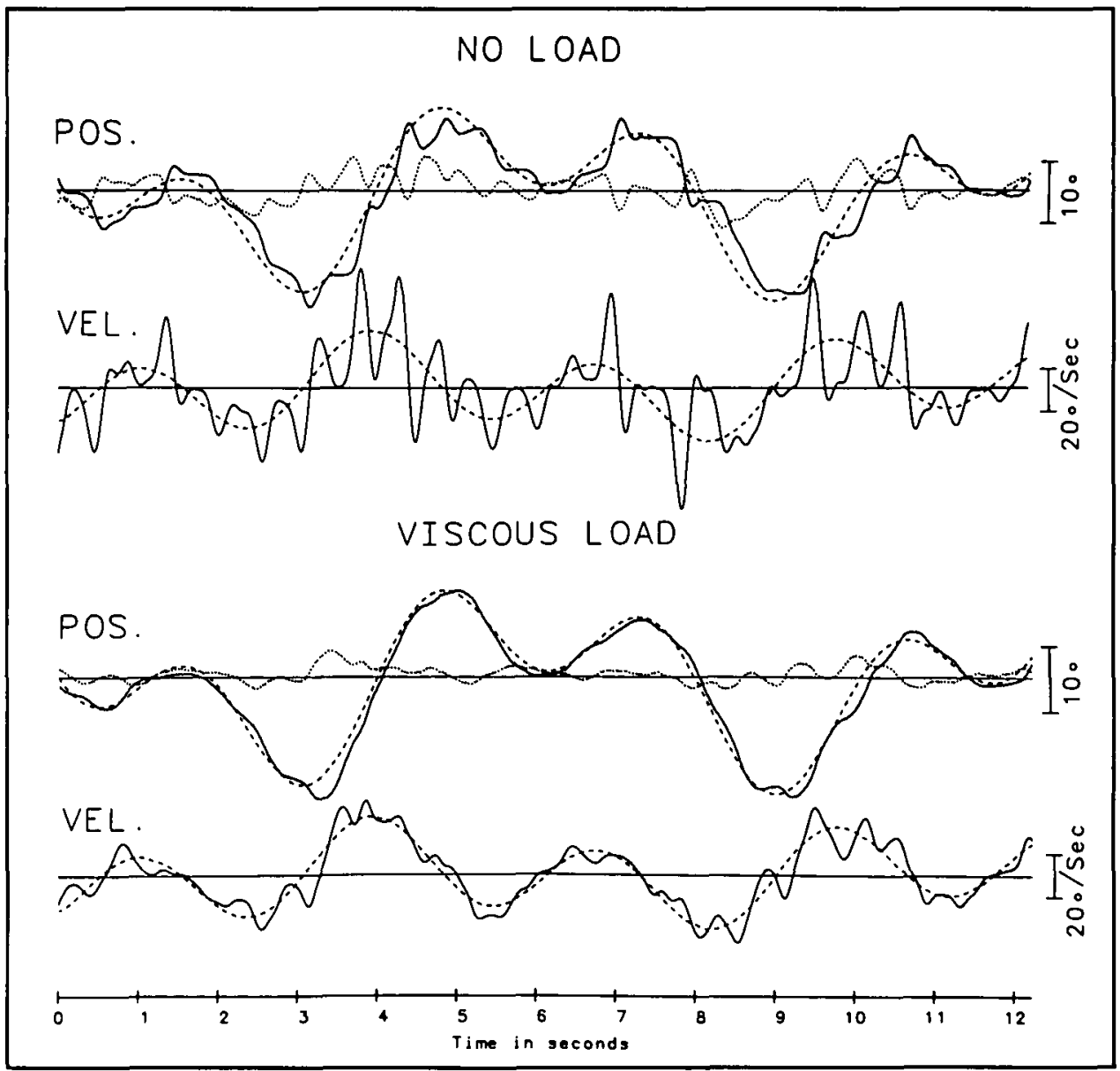

Figure 4 Effect of viscous loading on tracking performance in a patient with unilateral cerebellar incoordination following removal of a posterior fossa epidermoid tumour. The upper half of the figure show's position and velocity traces while the subject is tracking without any added load. The lower traces show the subject performing the same task against a viscous load of 0.44 Newtons/sect degree, provided by feeding back movement velocity to the torque motor coupled to the manipulandum. 
produced a significant decrease in positional error score for the clinically affected arms $(p=0.024)$. In the normal subjects the error score was increased with addition of an inertial load $(p=0.031)$. Figure 5 summarizes the results for one complete experiment on a patient with relatively mild unilateral arm incoordination following removal of a cerebellar hemangioblastoma. Positional error scores are shown for unloaded trials and for each of the six different loading conditions. The order of the bars (from left to right) indicates the sequence in which the conditions were presented to the subject. During unloaded trials the error scores were more than twice those obtained from control subjects. Addition of inertial loads did not result in any significant change in error score. However, with both levels of increased viscous stiffness and with the larger of the two levels of elastic stiffness used, the positional error score was reduced to about half of its unloaded value. Although the results shown in Figure 5 suggest a trend towards increasing error scores in the later unloaded trials for this particular patient, the initial ANOVA procedure showed that within each data group there was no difference in error scores between the seven sets of unloaded trials.

Table 3 summarizes the results obtained from all patients and control subjects using the six different loads. Tracking performance was considered to be "better" or "worse" if the mean positional error score obtained with the added load differed from results during unloaded trials with $\mathrm{p}$ value $<0.05$ (Student's t-test). Performance with the affected arm was improved in all five patients by adding a viscous load. In most cases, an increase in viscous stiffness did not cause a change in the performance of control subjects or in the clinically unaffected arms of the patients. Increased elastic stiffness did not improve performance in any of the control subjects and there were no consistent changes in the patients (some showed improvement but others had worse performance). No improvement was seen in either patients or controls with increased inertial load, and several subjects showed deterioration in tracking performance when weights were added to the hand.

The beneficial effects of viscous loading were also reflected in the velocity tracing (Figure 4). The velocity error score was reduced in all patients and there was also a reduction in the number of high velocity peaks and in the number of zero crossings. Increased stiffness and inertial load did not produce any consistent changes in velocity error scores, velocity peaks, or zero crossings.

\section{Time lag between target and hand movement}

It is possible that the large error scores produced by the cerebellar patients could be partially accounted for by delays in visuomotor processing causing an increased time lag between target and cursor movement. In all our patients as well as in control subjects, the hand movement usually lagged behind the target movement by an appreciable time (e.g. see Figure 1). Visual reaction times are known to be prolonged in patients with cerebellar dysfunction, 2,3 and this was confirmed for the type of motor task used in our study. Mean reaction time from movement of the visual target to onset of movement at the wrist joint was $336 \mathrm{msec}$. in the affected arms of the patients compared to $258 \mathrm{msec}$. for control subjects.

The method used to calculate the average tracking delay is illustrated in Figure 6. The abscissa represents the imposed time shift between target and cursor position. For the control subject in Figure 6 the cumulative error score during actual performance

\begin{tabular}{|c|c|c|c|}
\hline & Better & No Change & Worse \\
\hline \multicolumn{4}{|l|}{ Moderate Viscous Stiffness } \\
\hline Patients $\longleftarrow$ Affected Arm & $1,2,3,4,5$ & & \\
\hline Patients Clinically Unaffected Arm & 5 & $1,2,3,4$ & \\
\hline Control Subjects & 6 & $7,8,9,10,11$ & \\
\hline \multicolumn{4}{|l|}{ High Viscous Stiffness } \\
\hline Affected Arm & $1,2,3,4,5$ & & \\
\hline Patients $\angle$ Clinically Unaffected Arm & 1,5 & $2,3,4$ & \\
\hline Control Subjects & 7,8 & $6,9,10,11$ & \\
\hline \multicolumn{4}{|l|}{ Moderate Elastic Stiffness } \\
\hline Patients $~$ Affected Arm & 1,4 & $2,3,5$ & \\
\hline Clinically Unaffected Arm & 4,5 & & 1,2 \\
\hline Control Subjects & & $6,7,8,9,10,11$ & \\
\hline \multicolumn{4}{|l|}{ High Elastic Stiffness } \\
\hline Patients $\simeq$ Affected Arm & $1,2,3$ & 4 & 5 \\
\hline Control Subiects & & $1,3,4,5$ & \\
\hline \multicolumn{4}{|l|}{ Moderate Inertia } \\
\hline Affected Arm & & $1,2,3,5$ & 4 \\
\hline Patients Clinically Unaffected Arm & & 1,3 & $2,4,5$ \\
\hline Control Subjects & & $6,8,9,10,11$ & 7 \\
\hline \multicolumn{4}{|l|}{ High Inertia } \\
\hline Patients $\longleftarrow$ Affected Arm & & $1,2,4,5$ & 3 \\
\hline Pattents $\simeq$ Clinically Unaffected Arm & & & $1,2,4$ \\
\hline Control Subjects & & 9,11 & $6,7,8,10$ \\
\hline
\end{tabular}

Summary of effects of adding mechanical loads on tracking performance in all patients and control subjects. Each number represents one subject. Number 1-5 correspond to the patient numbers in Table 1. Numbers 611 represent control subjects. Scores in the better or worse columns mean that positional error score with the added load differed from control trials with $\mathrm{p}$ values $<0.05$ using a Student $\mathrm{t}$-test. 
of the task in this trial was 27530 . The curve in the figure represents the changing error scores which were calculated following sequential time shifts of cursor position with respect to target position. In this example the error score was minimized to a value of 23638 by shifting the cursor position $\pm 40 \mathrm{msec}$. The right half of Figure 6 shows similar calculations for one of the cerebellar patients. In this example a time shift of $\pm 100 \mathrm{msec}$ was required to minimize the error score.

Results of these calculations for all the subjects are summarized in Table 4. The mean time lag for the affected arm of the cerebellar patients was $98 \mathrm{msec}$. The corresponding value for control subjects was $55 \mathrm{msec}$. Although time shifts caused a greater absolute reduction in error score in cerebellar patients than in normal subjects, the errors still remained well above normal values, indicating that the problem was not simply due to an increased delay in tracking the target.

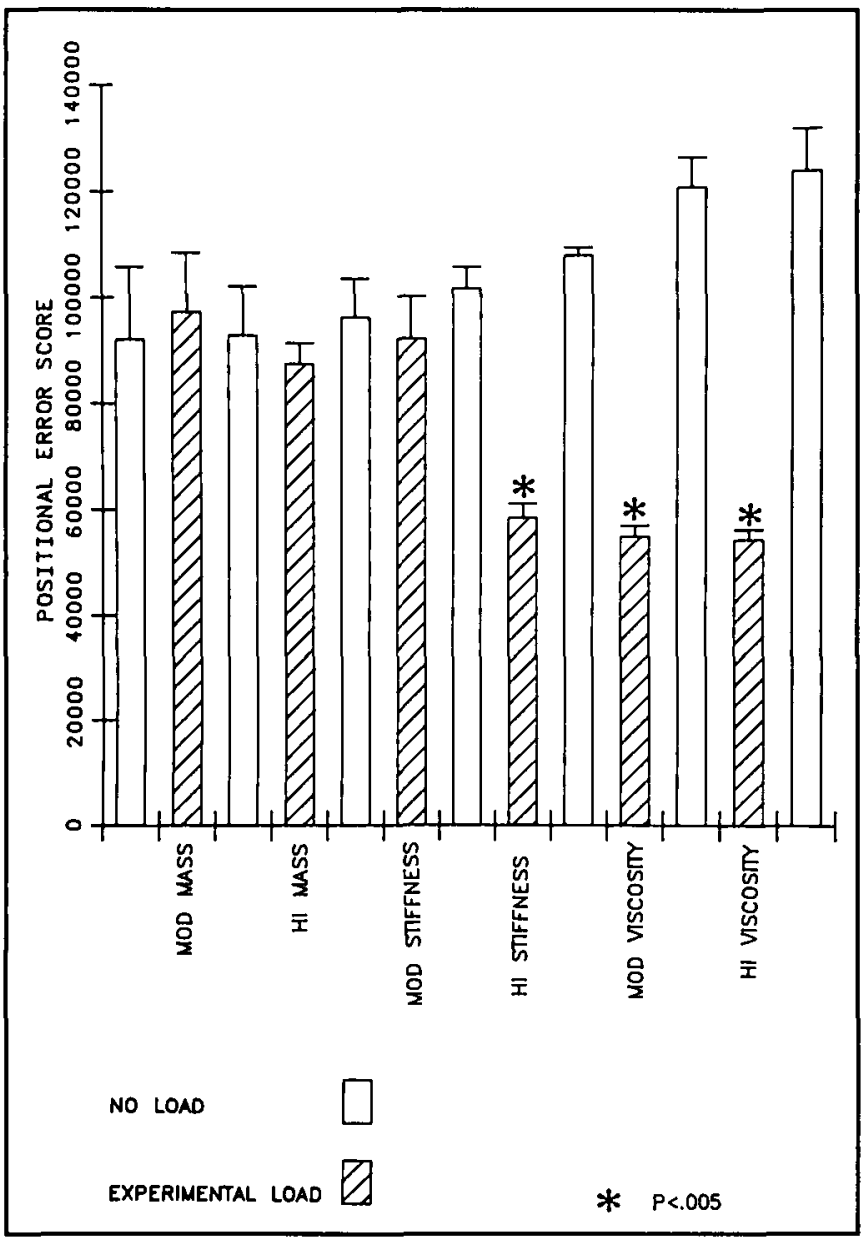

Figure 5 - Effects of different types of loading on the tracking performance of a patient with mild dysmetria following removal of a cere. bellar hemangioblastoma. The open bars represent mean positional error scores ( \pm standard errors) for three 20 second tracking trials without added load. The hatched bars are mean error scores for six trials with the mechanical load indicated on the abscissa. Each group of loaded trials was compared to the groups of control trials immediately preceding and following the loaded trials using a Student'st-test.

\section{Effects of eliminating visual feedback}

The inherent delays in tracking outlined above may cause the cerebellar patients to generate inappropriately rapid movements in an attempt to correct for visually detected errors and catch up to the target. To investigate this possibility we compared wrist movements from subjects tracking moving in a sinusoidal pattern, with similar movements performed by the subjects following removal of visual feedback of both the target and the cursor.

In all the cerebellar patients tested in this part of the study, the wrist position trace showed fewer irregularities after visual feedback was removed (Figure 7). Even more striking was a smoothing out of the velocity trace and a reduction in the numerous high velocity peaks observed when the subject was attempting to track the target.

\section{Discussion}

These results confirm that patients with cerebellar dysfunction attempting to track a slowly moving target have difficulty controlling the velocity of their arm movement. In comparison to normal subjects, they show frequent accelerations and decelerations as well as numerous high velocity peaks. Increasing the viscous load against which the subjects moved suppressed the velocity peaks and reduced tracking error. Similar increases in viscous load did not improve the tracking performance of control subjects or of the cerebellar patients using the clinically unaffected arm.

Tracking performance in patients with cerebellar dysfunction has also been studied by Beppu et al. ${ }^{3}$ Their subjects were required to make elbow flexion movements to track a target moving in a simple ramp pattern. Reaction times following onset of target movement were prolonged and variable. The subjects had difficulty generating movements with appropriate peak velocities during the initial "catch up phase" and were unable to maintain consistent movement velocity. The velocity records were characterized by an irregular "saccadic" pattern.

Miall et al ${ }^{8}$ also observed an increase in peak velocity of tracking movements in monkeys with reversible inactivation of the cerebellum produced by localized cooling. Their published recordings from the monkeys look remarkably similar to our recordings from human patients with cerebellar lesions. In a previous study by the same group it was concluded that intact monkeys use positional error to track unpredictable targets. When the target moves in a predictable sinusoidal pattern, target velocity becomes the main cue for tracking. With inactivation of the cerebellum the monkeys seem to revert to a situation where they are no longer able to utilize target velocity and become dependent on positional error for tracking even predictable targets.

Table 4: Effects of shifting cursor position with respect to target position to minimize positional error and determine tracking delay (see Figure 6).

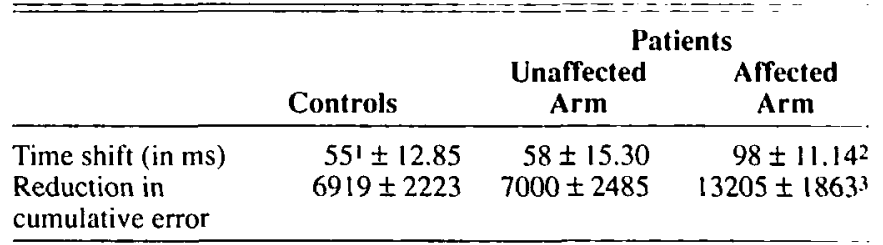

1 Mean values $\pm \mathrm{SE}$

$2 \mathrm{p}<0.025$

${ }^{3} \mathrm{p}<0.10$ 


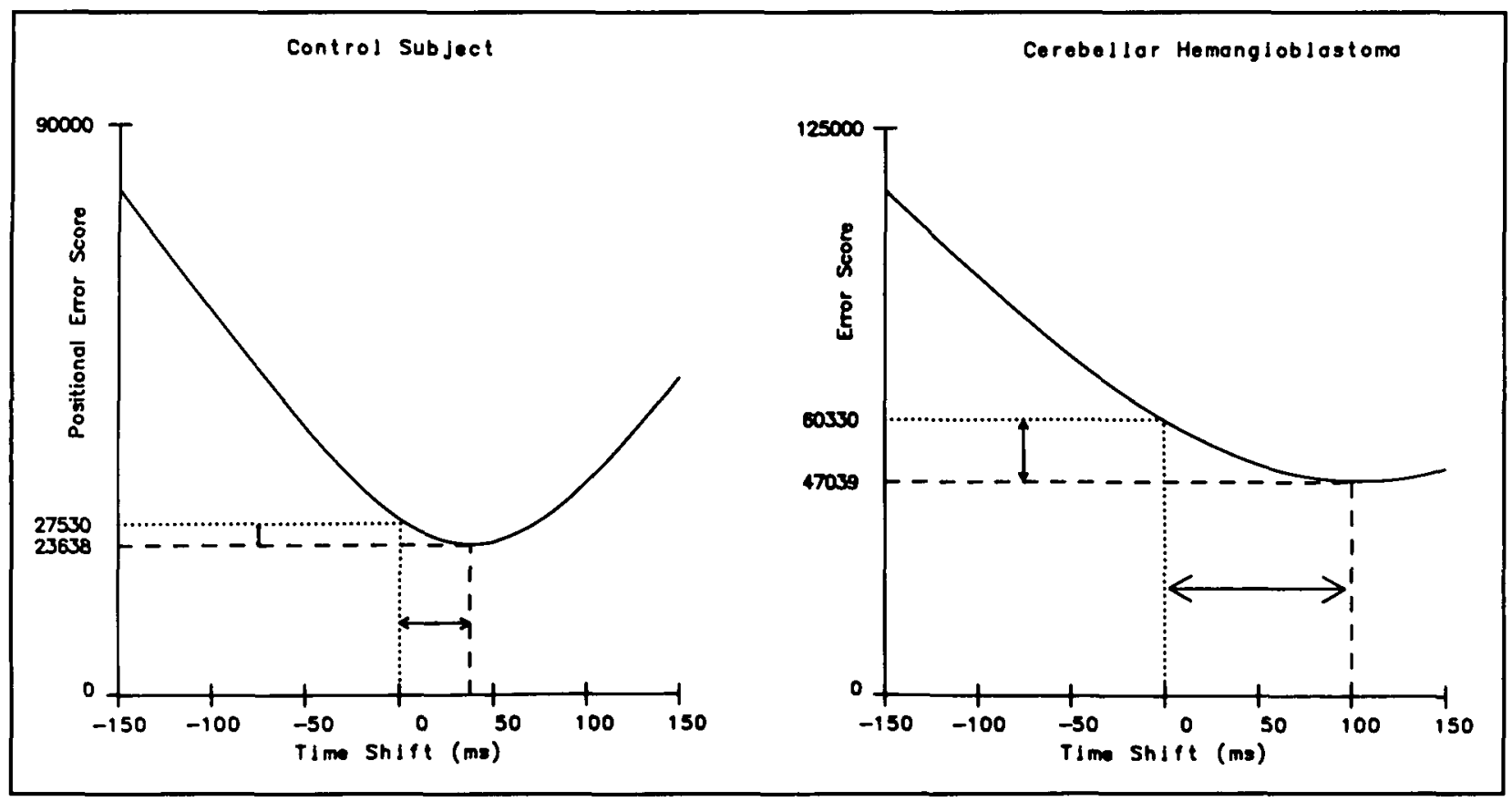

Figure 6-Illustration of method used to calculate the phase lag or tracking delay. Representative results are shown for a control subject and for a patient with cerebellar incoordination. In each case, the tracing representing cursor position was shifted horizontally with respect to the target position. This manoeuvre was performed sequentially in 10 msec steps and a hyperbolic curve was fitted to the data points representing error scores after each baseline shift. The amount of time shift required to minimize positional error score was defined as tracking delay. This was significantly greater in cerebellar patients than in control subjects.

In a recent study Sanes et al ${ }^{5}$ examined the effects of different types of mechanical loads on postural and kinetic tremor in patients with cerebellar lesions. They used viscous loads, constant loads opposing movement, and inertial loads and found that all three types of loading suppressed tremor. Our results differed in that only viscous loads consistently improved tracking performance. We did not test the effect of constant loads, and inertial loads had either no effect or, in some cases, actually worsened performance. These apparent discrepancies may be due to the fact that different types of patients were used in the two studies. In our patients the major abnormality was dysmetria with little or no associated tremor, whereas Sanes et al ${ }^{5}$ studied patients with prominent cerebellar tremor. Different mechanisms may be responsible for cerebellar tremor and dysmetria, and it may not be surprising that mechanical loading affects these two manifestations of cerebellar dysfunction in different ways.

\section{Hypotonia and cerebellar dysmetria}

Hypotonia was considered by Holmes to be one of the fundamental abnormalities following acute cerebellar injuries. ${ }^{2}$ In a strict sense hypotonia may be defined as the resistance felt by an examiner during passive manipulation of a limb or stretch of a muscle with the subject in a fully relaxed state. This resistance results from the combined effects of muscle stretch reflexes and the passive visco-elastic properties of the limb. Using this narrow definition only 2 of our 5 patients had definite hypotonia on clinical examination. However, Holmes has pointed out that hypotonia is not always easy to detect clinically, particularly in patients with long-standing cerebellar disorders. Furthermore the manifestations of hypotonia may be more apparent during active voluntary movement or maintenance of certain postures than during passive manipulation of the limbs.
Under normal conditions muscle tone may provide sufficient viscous resistance to dampen oscillations and irregularities during voluntary movements. Loss of this damping effect as a result of hypotonia could account for some of the abnormalities which are observed in cerebellar incoordination. Addition of an external viscous load might restore some of the damping which is provided by normal muscle tone.

\section{Mechanical considerations}

The characteristics of a movement are determined not only by the motor commands which generate the movement but also by the mechanical properties of the limb being moved. Changes in the mechanical properties of a limb have been shown to modify cerebellar tremor in monkeys ${ }^{10}$ and in humans. ${ }^{11}$

These properties include elastic stiffness, viscous stiffness and inertia. To a good approximation a limb behaves like a set of masses, springs and dashpots, and its characteristics can be modelled by a second order linear system. ${ }^{12}$ A simple mass-spring system will develop oscillations in response to a perturbation, but in all real systems there is some damping which causes the oscillations to decay.

The relationship between these variables can be expressed by the following equation:

$$
\zeta=\frac{1}{2} \times \sqrt{K \times M}
$$

where $\zeta$ is the damping factor, $\beta$ represents viscosity of the dashpot, $M$ represents mass, and $K$ is the stiffness of the spring (See Stein and Lee). ${ }^{13}$

From this equation it is apparent that reduced viscosity will result in decreased damping. Adding viscosity should increase damping and suppress oscillations. It would also be predicted 


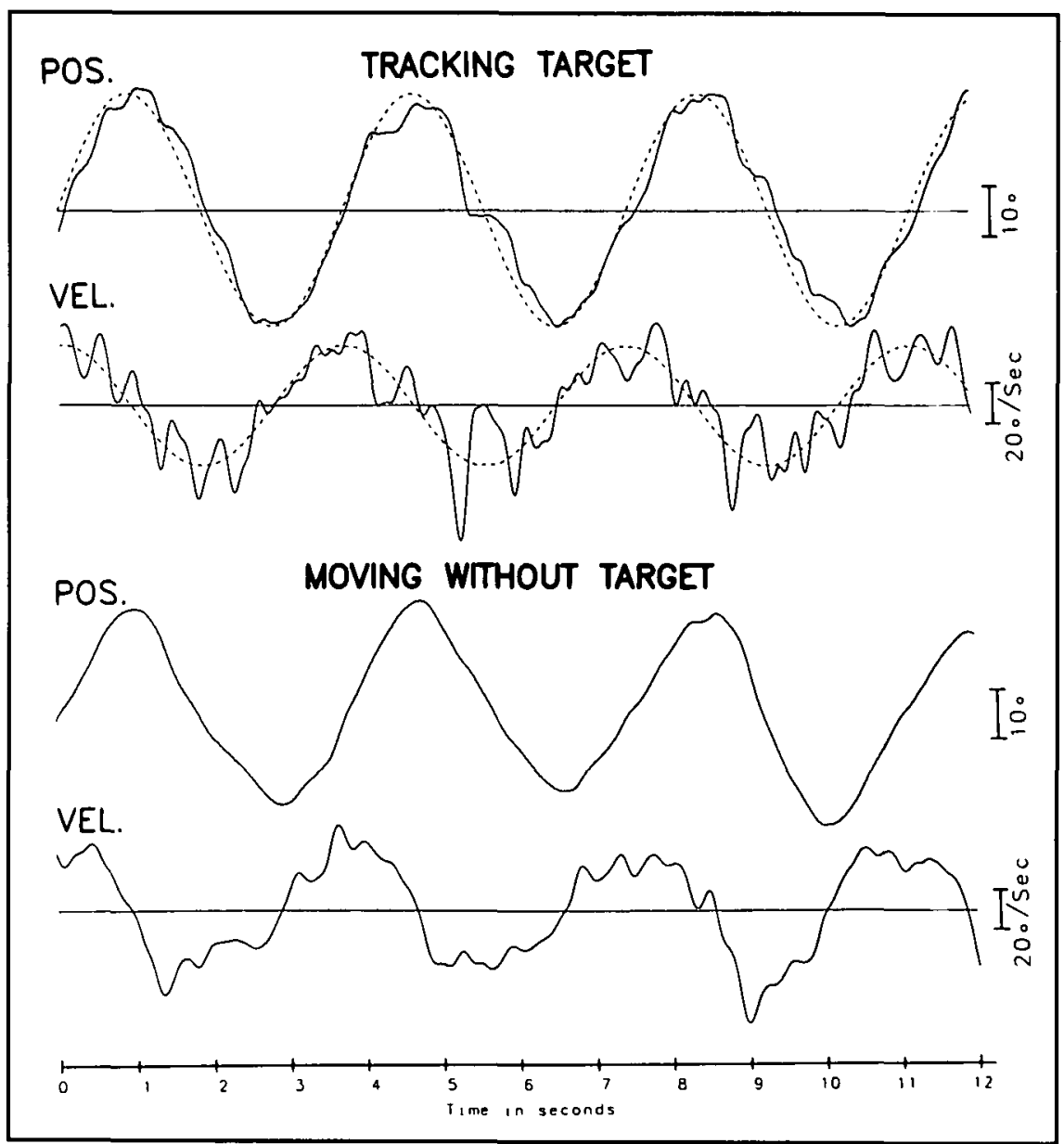

Figure 7 - Effect of removing visual feedback from a patient with cerebellar incoordination. The top traces show wrist position and velocity while the subject is tracking a target moving in a sinusoidal pattern. The bottom half of the figure shows position and velocity with the subject attempting to reproduce the same pattern of movement afier removal of the display showing target and cursor position.

from this equation that increasing the stiffness or the mass of the system could result in decreased damping. This might account for the observation that the addition of inertial loads worsened tracking performance in some of our subjects.

The values for viscous and elastic stiffness used in our experiments were selected arbitrarily. Theoretically, it should be possible to calculate the damping factor for a limb and to use the above equation to select the most appropriate combination of elastic and viscous stiffness required to restore critical damping in an individual patient.

\section{Co-activation of antagonistic muscles and stiffness}

Under normal conditions, co-activation of opposing groups of muscles may be one mechanism for internally increasing limb stiffness during performance of skilled motor tasks. Akazawa et al ${ }^{14}$ showed that stretch-evoked stiffness in the human thumb increased linearly with the level of co-activation of flexor and extensor muscles. Co-activation of opposing muscles in the human forearm causes an increase in both stiffness and viscosity of the limb. ${ }^{12}$ Hasan $^{15}$ has reported that the joint stiffness resulting from increased activation of agonists and antagonists plays an important role in decreasing the amount of perceived effort during the task.
It has been shown that monkeys tend to co-activate wrist flexors and extensors while they are learning a new tracking task. ${ }^{16}$ It has also been proposed that the cerebellum plays an important role in switching patterns of motor activation from reciprocal inhibition to co-contraction, depending on the type of task being carried out. ${ }^{17}$

If co-activation is used to produce the internal viscous stiffness required to carry out precisely controlled tracking movements, one might predict that there would be less co-activation than normal in cerebellar patients performing tracking tasks. There are very few studies which have examined this possibility, but Beppu et al $^{3}$ actually found increased levels of co-activation in their patients with cerebellar dysfunction.

\section{Role of sensory feedback}

The exact role which the lateral cerebellum plays in control of limb movements is still not fully understood. One theory proposed by several investigators (see Brooks and Thach) ${ }^{7}$ considers that the cerebellum functions as a comparator. Efference copy of motor commands is sent to the cerebellum where comparisons are made with proprioceptive feedback from the periphery indicating what is actually occurring during the movement. If errors are detected then corrective signals are transmitted to 
motor cortex. Although this is an oversimplified view of cerebellar function which may not fit entirely with current knowledge concerning inputs to specific areas of cerebellar cortex, it may help to partially explain some of the errors in voluntary movement which occur following cerebellar damage.

The cerebellum also receives visual information from extrastriate visual cortex ${ }^{18}$ and some of the irregularities and oscillations occurring during visual tracking tasks like those performed by our subjects could be interpreted as inappropriate or excessive corrections for visually detected errors. ${ }^{4}$ If this is true, it might be predicted that patients with cerebellar lesions could produced smoother movements without visual guidance. There is very little evidence from clinical observations that this is the case for reaching or pointing movements involving an entire limb. However, with the more simple single joint tracking movements used in our study, there was a smoothing of irregularities and a reduction in number of high velocity peaks when visual feedback was removed and subjects were asked to reproduce the same movements without vision.

Other investigators have shown similar smoothing of irregularities of movement when visual feedback of the target and limb position is removed. 4.5.19 However, somewhat different conclusions were reached from experiments on monkeys by Flament et al. ${ }^{20}$ They observed that withdrawal of visual feedback failed to alter cerebellar tremor and concluded that proprioceptive feedback associated with activation of stretch reflexes was a major factor in driving the tremor.

Delays in visuomotor processing may be partially responsible for abnormal responses to visually detected errors. Visual reaction times are prolonged in patients with cerebellar dysfunction. ${ }^{2,3}$ Our recordings showed that the time lag or tracking delay in the affected arms of the patients was significantly prolonged in comparison to their normal arms and to results from control subjects. The significance of these tracking times lags is uncertain. If subjects were able to predict target movement with complete accuracy, then the time lag (and the error score) should drop to values near zero after a sufficient number of practice trials. On the other hand, if the patients were totally dependent on visual feedback for tracking the target, one might expect time lags with values similar to the visual reaction time. The values obtained in both patients and control subjects lay between these two extremes, suggesting that this particular tracking task is performed by combining strategies of predicting target movement and reacting to visually detected changes in target position.

\section{Possible clinical implications}

Conventional rehabilitation techniques are of limited value for patients with cerebellar dysfunction. Intention tremor may be diminished by adding weights to the limb.21.22 However, addition of weights to the hand did not improve tracking performance and in some cases actually caused an increase in tracking error in our patients, where the major deficit was dysmetria rather than intention tremor.

It remains to be shown whether motor performance for whole limb movements by patients with cerebellar dysfunction can be improved by increasing viscous resistance, as was our finding for simple movements at a single joint with the arm supported to eliminate gravitational effects. Several approaches might be considered to develop viscous interfaces to help improve functional activities. One type of application might consist of increasing the viscous stiffness of devices currently used by patients such as environmental control systems, feeding devices, or joy sticks used to control powered wheelchairs. Another possibility might include the design of a garment or orthosis with inherent viscous stiffness to provide a "tuned" mechanical compliance against which the upper limb can be moved smoothly during voluntary activity.

\section{ACKNOWLEDGEMENT}

This work was supported by grants from the Medical Research Council of Canada, the Alberta Heritage Foundation for Medical Research and the Physiotherapy Foundation of Canada. The authors express their appreciation to Mr. David White who provided invaluable technical assistance and computer programming.

\section{REFERENCES}

1. Holmes G. The symptoms of acute cerebellar injuries due to gunshot injuries. Brain 1917; 40: 461-535.

2. Holmes $\mathrm{G}$. The cerebellum of man. Brain 1939; 62: 1-30.

3. Beppu H, Suda M, Tanaka R. Analysis of cerebellar motor disorders by visually-guided elbow tracking movements. Brain 1984; 107: 787-809.

4. Beppu H, Nagaoka M, Tanaka R. Analysis of cerebellar motor disorders by visually-guided elbow tracking movements: 2 . Contribution of the visual cues on slow ramp pursuit. Brain 1987; 110: 1-18.

5. Sanes JN, LeWitt PA, Mauritz KH. Visual and mechanical control of postural and kinetic tremor in cerebellar system disorders. J Neurol Neurosurg Psychiatry 1988; 51: 934-943.

6. Zee DS, Yee RD, Gogan DG, et al. Ocular motor abnormalities in hereditary cerebellar ataxia. Brain 1976; 99: 207-234.

7. Brooks VB, Thach WT. Cerebellar control of posture and movement. In: Brookhart, Mountcastle, Brooks, Greiger, eds. Handbook of Physiology, Section 1, The Nervous System. American Physiological Society. (Bethesda, Maryland) 1981; 877-946.

8. Miall RC, Weir DJ, Stein JF. Visuo-motor tracking during reversible inactivation of the cerebellum. Exp Brain Research 1987; 65: 455-464.

9. Miall RC, Weir DJ, Stein JF. Manual tracking of visual targets by trained monkeys. Behav Brain Res 1986; 20: 185-201.

10. Vilis T, Hore J, Effects of changes in mechanical state of limb on cerebellar intention tremor. J Neurophysiol 1977; 40: 1213-1224.

11. Homberg V, Hefter H, Reiners K, et al. Differential effects of changes of mechanical limb properties on physiological and pathological tremor. J Neurol Neurosurg Psychiatry 1987; 50: 568-579.

12. Lacquaniti F, Licata F, Soechting JF. The mechanical behavior of the human forearm in response to transient perturbations. Biol Cybern 1982; 44: 35-46.

13. Stein RB, Lee RG. Tremor and clonus. In: Brookhart, Mountcastle, Brooks, Geiger, eds. Handbook of Physiology, Section 1. The Nervous System, chap. 9. American Physiological Society. (Bethesda, Maryland) 1981; 325-343.

14. Akazawa K, Milner TE, Stein RB. Modulation of reflex EMG and stiffness in response to stretch of human finger muscle. $J$ Neurophysiol 1983; 49: 16-27.

15. Hasan Z. Optimized movement trajectories and joint stiffness in unperturbed, inertially loaded movements. Biol Cybern 1986; 53: 373-382.

16. Schieber MH, Thach WT. Trained slow tracking. II. Bidirectional discharge patterns of cerebellar nuclear, motor cortex, and spindle afferent neurons. J Neurophysiol 1985; 54: 1228-1270.

17. Smith AM. The coactivation of antagonist muscles. Can J Physiol Pharmacol 1981; 59: 733-747.

18. Glickstein M, May JG III, Mercier BE. Corticopontine projection in the macaque: the distribution of labelled cortical cells after large injections of horseradish peroxidase in the pontine nuclei. $J$ Comp Neurol 1985; 235: 343-359. 
19. Miller RG, Freund HJ. Cerebellar dyssynergia in humans - a quantitative analysis. Ann Neurol 1980; 8: 574-579.

20. Flament D, Vilis T, Hore J. Dependence of cerebellar tremor on proprioceptive but not visual feedback. Exp Neurol 1984; 84: 314-325.

21. Hewer RL, Cooper R, Morgan MH. An investigation into the value of treating intention tremor by weighting the affected limb. Brain 1972; 95: 579-590.
22. Morgan MH, Hewer RL, Cooper R. Application of an objective method of assessing intention tremor - a further study on the use of weights to reduce intention tremor. J Neurol Neurosurg Psychiatry 1975; 38: 259-264. 